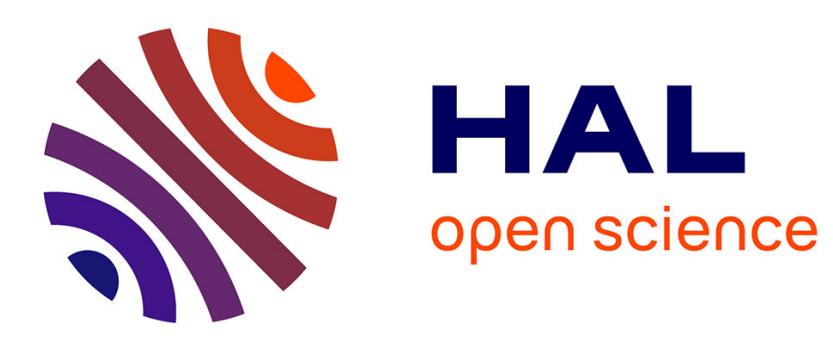

\title{
Du parlementarisme minoritaire régulé au parlementarisme minoritaire démembré. Le legs de la crise ministérielle suédoise survenue à l'issue des élections législatives de septembre 2018
}

Laurent Léothier

\section{To cite this version:}

Laurent Léothier. Du parlementarisme minoritaire régulé au parlementarisme minoritaire démembré. Le legs de la crise ministérielle suédoise survenue à l'issue des élections législatives de septembre 2018. Revue française de droit constitutionnel, 2019, 118, pp.357-363. 10.3917/rfdc.118.0357 . halshs02560746

\author{
HAL Id: halshs-02560746 \\ https://shs.hal.science/halshs-02560746
}

Submitted on 2 May 2020

HAL is a multi-disciplinary open access archive for the deposit and dissemination of scientific research documents, whether they are published or not. The documents may come from teaching and research institutions in France or abroad, or from public or private research centers.
L'archive ouverte pluridisciplinaire HAL, est destinée au dépôt et à la diffusion de documents scientifiques de niveau recherche, publiés ou non, émanant des établissements d'enseignement et de recherche français ou étrangers, des laboratoires publics ou privés. 


\author{
Laurent LÉOTHIER \\ Docteur d'État en droit public, ILF-GERJC \\ CNRS UMR7318 (DICE), Aix-Marseille Université
}

\title{
Du parlementarisme minoritaire régulé au parlementarisme minoritaire démembré Le legs de la crise ministérielle suédoise survenue à l'issue des élections législatives de septembre 2018
}

Cent trente-quatre jours! Voici ce qu'il aura fallu pour sortir la Suède de la plus longue crise ministérielle de son histoire récente. Pourtant connue pour son consensus politique érigé au rang de dogme national, la Suède semble à son tour touchée par les soubresauts institutionnels et politiques qui traversent l'Europe. Bien plus qu'une tempête passagère, c'est l'effondrement du système de gouvernement minoritaire configuration institutionnelle à laquelle recourent fréquemment les pays scandinaves qui semble marquer la conclusion de cette période de crise ouverte par les élections législatives de septembre 2018. En renouvelant le Riksdag, unique chambre du Parlement, les Suédois ont, par la diversité de leurs opinions et la complexité d'un système électoral toujours en proie à l'hésitation entre scrutin majoritaire et proportionnel, conduit leur régime dans une impasse politique inédite. Pourtant en décembre 2014, tout avait été fait pour structurer un parlementarisme minoritaire sérieusement ébranlé par la poussée et l'établissement durable de l'extrême droite (les Démocrates de Suède ${ }^{1}$ ) au Parlement. La crise politique à l'époque ${ }^{2}$ avait débouché sur un accord audacieux devant durer jusqu'en 2022 3 (c'est-à-dire sur les deux prochaines législatures du Riksdag) qui engageait six des huit partis politiques représentés au Parlement. Sorte de code de bonne conduite, l'accord complète les dispositions constitutionnelles en précisant les usages concernant la formation des gouvernements dits minoritaires, c'est-à-dire ceux dont l'investiture, la composition et le soutien à long terme, ne permettent pas d'atteindre la majorité absolue des suffrages à la chambre unique du Parlement.

1 En suédois : Sverigesdemokraterna(SD)

2 En décembre 2014, le gouvernement conduit par le social-démocrate Stefan Löfven s'est retrouvé contraint d'exécuter un budget qui n'est pas celui proposé par le gouvernement. En Suède, il est en effet possible pour l'opposition de proposer une loi de finances concurrente à celle du gouvernement. L'extrême-droite avait, à la surprise générale, rallié ses voix à la coalition de centredroit pour faire adopter le budget concurrent. Le Premier ministre avait alors menacé de dissoudre le Parlement afin que les électeurs tranchent le conflit.

3 L'accord de décembre (Decemberöverenskommelsen) dit DÖ conclu par le parti Social-Démocrate, Socialdemokraterna (S), et le parti Écologique, Miljöpartiet - De Gröna (MP) avec la coalition de centre-droite dite L'Alliance (Alliansen) regroupant les Modérés, Moderaterna (M), le parti du centre, Centerpartiet (C), les Libéraux, Folkpartiet (FP) rebaptisé depuis Liberalerna (L) et les Chrétien-Démocrates, Kristdemokraterna (KD), avait comme objectif de réduire l'influence des deux partis d'extrême, le parti de Gauche, Vänsterpartier (V), et les Démocrates de Suède dans les débats parlementaires. L'accord prévoit que la coalition arrivée en tête forme le gouvernement et le chef du principal parti de celle-ci en devienne le Premier ministre. La coalition défaire lors du scrutin s'engage à ne pas voter contre lors de l'élection du candidat à la chefferie du gouvernement et à ne pas s'opposer à l'adoption du budget du pouvoir exécutif. V. E. MATUTANo, « Le legs de la crise politique suédoise du mois de décembre 2014 », RFDC, n²016/2, pp. 357 - 366. 
Or les élections législatives de septembre 2018 et la crise ministérielle qui en découle marquent la fin de la politique des blocs ${ }^{4}$ au Parlement qui était la pierre angulaire du fonctionnement du parlementarisme minoritaire. Ainsi, cet échec d'un retour à un équilibre du parlementarisme minoritaire de surcroît renforcé par un accord (I) marque l'avènement d'un parlementarisme démembré inhabituel (II) dans ce pays où y règne un système de confiance présumée des députés à l'égard du gouvernement.

\section{I - L'échec du rétablissement d'un parlementarisme minoritaire des blocs}

L'élection législative de septembre 2018 fait apparaître un équilibre des forces quasi parfait entre les deux blocs ${ }^{5}$, ainsi que l'installation durable de l'extrême-droite au Parlement ${ }^{6}$. Ce paysage politique partitionné rend impossible la constitution d'un gouvernement minoritaire autour d'un bloc (A), ce qui marque l'étiolement de la structuration binaire des combinaisons parlementaires (B).

\section{A - L'impossible constitution d'un gouvernement minoritaire autour des blocs}

Lors de la parution des résultats définitifs, il n'y avait guère de doute, la Suède s'orientait vers le processus de désignation du gouvernement le plus complexe de son histoire. Pourtant en décembre 2014, les six partis de gouvernement s'accordent sur une solution politique inédite pour enrailler l'influence de l'extrême-droite sur la vie parlementaire. Le principal atout de cet accord est le complément des dispositions constitutionnelles en matière de désignation du Premier ministre. Lacunaire, la Constitution suédoise (Regeringsformen ${ }^{7}$ ) se contente d'inscrire le pouvoir d'élection du Riksdag pour le poste de Premier ministre. La formulation l'article en question ne consacre pas un véritable pouvoir d'investiture du Parlement, en ce sens qu'il ne s'agit pas d'un vote de confirmation - il n'est pas nécessaire que le candidat réunisse une majorité favorable à son élection - mais plutôt d'un droit de veto du Riksdag, la candidature ne pouvant être rejetée qu'à la majorité absolue des membres du Parlement (soit cent soixante-quinze voix) ${ }^{8}$. Pourtant, il est de coutume que le nombre d'abstention des éventuels groupes parlementaires soutenant l'action du gouvernement sans y participer suffit à écarter le seuil fatidique de la majorité absolue. L'accord de décembre 2014 érige cette attitude politique en une sorte de code de bonne conduite du bloc vaincu lors du scrutin. Ainsi, selon les termes de ce même accord, le bloc réunissant le

4 La Suède est l'archétype de la division en deux blocs de la vie politique. D'un côté, l'on trouve le bloc dit bourgeois (borgeliga block) regroupant les formations politiques de droite et du centre (M, KD, $\mathrm{C}$ et $\mathrm{L}$ ) et d'un autre côté le bloc rouge-vert associant les Sociaux-démocrates et les écologistes auquel s'ajoute le soutien du parti de Gauche. Le bloc bourgeois s'est regroupé depuis 2006 sous l'étiquette de l'Alliance (Alliansen) alors que l'entente entre les verts et les sociaux-démocrates reste plus libre.

5 Le bloc de gauche, en comptant les députés du parti de Gauche, totalise cent quarante-quatre élus alors que le bloc bourgeois en compte cent quarante-trois.

6 L'extrême-droite remporte soixante-deux sièges au Parlement soit son meilleur score depuis son entrée au Riksdag lors des élections législatives de 2010.

7 La Forme du gouvernement (Regeringsformen) est la principale composante de la Constitution suédoise réglant le fonctionnement des institutions. Les trois autres composantes sont la loi fondamentale sur la liberté d'expression (Yttrandefrihetsgrundlagen), la loi de succession du trône (Successionsordningen), et la loi sur la liberté de la presse (Tryckfrihetsförordningen)

8 Chap. VI, art. 2, al. 2 Regeringsformen 
plus grand nombre de parlementaires compose le gouvernement; le Premier ministre étant élu par celui-ci avec le soutien des abstentions d'au moins d'une partie des groupes parlementaires composant l'autre bloc. En suivant cette logique, le bloc rouge-vert (Rödgröna) doit à l'issue des élections, composé le gouvernement (il détient en effet une voix de plus que l'Alliance), mais l'étroitesse de la victoire ajoutée à la volonté d'une partie des Modérés de s'allier à l'extrême-droite enterrent les dispositions de l'accord. Le nouveau président du Riskdag, le Modéré Andreas Norlén, élu grâce au soutien des Démocrates de Suède, propose alors de suivre les conclusions de l'accord de décembre 2014 afin d'y mettre définitivement un terme. Le Premier ministre sortant, Stefan Löfven, est alors proposé pour le poste mais une majorité absolue de parlementaire le rejette $^{9}$. Ainsi puisqu'une majorité semble s'être formée entre la droite et l'extrêmedroite, le président du Parlement propose à Ulf Kristersson, président des Modérés, de mener des négociations en vue de la formation d'un gouvernement dont il prendrait la tête. Or avant même son élection, un revers d'alliance se produit au sein de l'Alliansen; les centristes et les libéraux refusent de le soutenir au poste de Premier ministre étant donné qu'il souhaite que son futur gouvernement minoritaire s'appuie sur le soutien des Démocrates de Suède. Ainsi, l'alternative incarnée par l'Alliance s'effondre lors de la seconde élection du Premier ministre, une majorité absolue - comprenant la gauche, les centristes et les libéraux - met un veto à Ulf Kristersson ${ }^{10}$. S'ouvre alors un étiolement de la structuration binaire des combinaisons parlementaires venant éprouver la solidité des institutions suédoises.

\section{B - L'étiolement de la structuration binaire des combinaisons parlementaires}

Les refus successifs du Riksdag des candidatures des deux primoministrables met en avant un second aspect du résultat de l'élection. Tous les partis politiques minoritaires des blocs, à l'exception des Verts qui paient leur participation à quatre années de gouvernement, sont en net progression par rapport aux Sociaux-démocrates et aux Modérés qui voient leur leadership décliner. Aussi, si l'accord de décembre 2014 cherche à empêcher les Démocrates de Suède d'être les faiseurs de roi, il reste silencieux sur le rôle des formations minoritaires dans la conduite des crises ministérielles. Ces dernières deviennent plus autonomes par rapport à la principale formation politique de leur bloc qui prêtant historiquement à la direction du gouvernement. On assiste alors à un déclin des alliances partisanes vieilles de plus de dix ans. Face à la volonté affichée des Modérés de composer un gouvernement d'Allians soutenu par l'extrême-droite, les centristes et les libéraux se désolidarisent. Une nouvelle série de missions d'informations est alors confiée alternativement à Stefan Löfven puis à Ulf Kristersson ${ }^{11}$ afin d'établir un gouvernement soutenu par ce nouveau bloc du centre devenu indispensable à l'élection du Premier ministre. Ainsi, toutes les combinaisons sont

9 Il s'agit de l'ensemble des élus de l'Alliance (M, C, KD, L) ainsi que les Démocrates de Suède. La majorité absolue de 175 est franchie en atteignant 204 votes négatifs. V. Voterignsprotokoll 2018/19 : Statsministeromröstning, 25 septembre 2018

10 L'opposition se manifeste par 195 voix contre. V. Voterignsprotokoll 2018/19: Statsministeromröstning, 14 novembre 2018

11 La complexité de la crise ministérielle apparaît dans le nombre important de missions d'information confiées par le président du Parlement aux chefs de parti : Stefan Löfven (S), $24-25$ sept. ; Ulf Kristersson (M), 2 - 14 oct. ; Stefan Löfven (S), 15 - 22 oct. ; Ulf Kristersson (M), 5 - 14 nov. ; Annie Lööf (C), 15 - 22 nov. ; Stefan Löfven (S), 23 nov. - 14 déc. ; Stefan Löfven (S), 11 - 18 janv. 
envisageables, les élus du parti de l'Environnement insistent d'ailleurs pour que la cheffe du parti du Centre, Annie Lööf, prenne la tête d'une nouvelle mission d'information. Chargée par le président du Parlement de mener les négociations, ses conclusions témoignent de l'effondrement de la conception binaire du parlementarisme suédois. Trois alternatives sont envisagées: un gouvernement de l'Allians soutenu par les sociaux-démocrates ; une grande coalition entre les Sociaux-Démocrates et les Modérés ; enfin un petit gouvernement du centre (små centern-regering) soutenu alternativement par la droite ou par la gauche ${ }^{12}$. Les conditions posées par les centristes au maintien de l'Allians ne trouvant aucun écho parmi ses membres, les amènent à s'éloigner des schémas politiques traditionnels et par conséquent à introduire les modalités d'un parlementarisme minoritaire démembré.

\section{II - L'avènement d'un parlementarisme minoritaire démembré}

L'échec de la formation d'un gouvernement minoritaire en suivant le schéma traditionnel des blocs conduit inéluctablement à renforcer la déconnexion entre la participation gouvernementale et le soutien parlementaire dont bénéficie l'exécutif $(A)$, ainsi qu'à déconstruire le système de confiance présumée (B).

\section{A - La déconnexion accentuée entre la participation et le soutien au gouvernement}

L'impossible retour à un gouvernement par les blocs et le rejet du compromis centriste d'un petit gouvernement $d u$ centre ouvre progressivement la voie au mécanisme de dissolution prévue par la Constitution ${ }^{13}$. Après quatre votes négatifs du Riksdag à l'occasion de l'élection du Premier ministre, la dissolution de la chambre est prononcée et des élections anticipées ont lieu afin de conduire de nouveaux députés pour clôturer la législature ${ }^{14}$. L'intérêt provoqué par de nouvelles élections serait de pouvoir faire correspondre la composition politique du prochain gouvernement au soutien qu'il recevrait au Parlement. Déjà le gouvernement démissionnaire de Stefan Löfven en place depuis septembre 2014 devait compter sur un soutien à géométrie variable, en ce sens qu'il était à direction social-démocrate, avec la participation du parti Écologiste, mais ne pouvait compter que sporadiquement sur le soutien du parti de Gauche ou de quelques députés de l'Allians. Or la fissure au sein de la coalition de centre-droit entre son pôle modéré (composé des centristes et des libéraux) et conservateur (formée des modérés et des chrétien-démocrates) accentue cette tendance à la discordance entre soutien et participation au gouvernement. La situation inédite

12 La Suède a connu un tel gouvernement entre octobre 1978 et octobre 1979 lorsque le libéral Ola Ullsten dirige un gouvernement du centre ultra-minoritaire ne disposant du soutien que de trenteneuf députés.

13 La probabilité d'une dissolution est de plus en plus plausible en raison d'un troisième rejet à la majorité absolue des députés à la candidature de Stefan Löfven proposée le 14 décembre. V. Voterignsprotokoll2018/19 : Statsministeromröstning, 14 décembre 2018

14 La dissolution et les élections extraordinaires (extraval) qui la suivent sont un phénomène extrêmement rare en Suède. La Constitution (chap. VI, art. 3 RF) prévoit que les députés élus lors d'un tel scrutin terminent le mandat initié par leurs prédécesseurs. Ainsi, la législature débutée à l'issue des élections ordinaires ne prend fin qu'à son terme régulier après quatre ans. La dernière dissolution du Riksdag remonte à avril 1958, la Suède était alors sous l'empire de la Constitution de 1809. 
dans laquelle se trouvent les négociations durant le mois de décembre conduit à une reconfiguration singulière du rôle du président du Riksdag dans la procédure de formation $\mathrm{du}$ gouvernement. Alors qu'il est d'ordinaire chargé de désigner l'informateur ${ }^{15}$ - qui n'est d'autre que le futur Premier ministre - pour mener les négociations, il est, cette fois, lui-même chargé d'une telle mission. Aussi, bien qu'il soit tenté d'avantager Ulf Kristersson, candidat de son parti politique d'origine, le président du Riksdag (talman) doit finalement se résoudre à faire appel à Stefan Löfven qui a gagné le ralliement des centristes et des libéraux à son gouvernement; actant ainsi la déconstruction du système de la confiance présumée.

\section{B - La déconstruction programmée du système de la confiance présumée}

En élisant Stefan Löfven comme Premier ministre une seconde fois à la tête d'un gouvernement minoritaire des deux tiers ${ }^{16}$ à l'issue d'un scrutin qui a vu les votes négatifs l'emporter, les députés suédois semblent faire table rase du principe fondamental de l'accord de décembre 2014, ainsi que du système de confiance présumée. Si jusqu'à présent les gouvernements minoritaires pouvaient soit prétendre au soutien majoritaire, soit bénéficier de l'abstention-approbation fondant «une présomption de confiance en faveur du gouvernement (ce qui) signifie qu'il appartient à l'opposition parlementaire de faire la preuve qu'elle est majoritaire ${ }^{17}$, il est désormais nécessaire de prouver le contraire. En effet, l'abstention des libéraux et des centristes lors de l'élection du Premier ministre ${ }^{18}$ ne garantit pas au gouvernement leur soutien sur le long terme. Cette éventualité est d'ailleurs sérieusement à prendre en compte depuis que les chefs de ces partis ont annoncé être dans une sorte d'opposition au gouvernement qu'ils ont contribué à faire élire. Aussi, si le premier volet de l'accord semble avoir volé en éclat, on peut se demander ce qu'il adviendra du second, à savoir l'absence d'obstacle à l'approbation du budget proposé par le gouvernement. Sur ce point, l'accord vise également à édicter un code de bonne conduite de l'opposition afin d'éviter le retour à une situation similaire à celle de décembre 2014, lorsque le gouvernement s'était vu contraint d'exécuter un budget qui n'était pas le sien. En somme l'abstention-approbation des centristes et des libéraux apparaît comme un acte de bonne volonté visant à éviter la dissolution, mais la reconstruction du paysage politique autour des blocs après un probable changement de ligne chez les conservateurs, allié à un partage original du pouvoir ${ }^{19}$ pourrait conduire à des mises en minorité fréquentes $\mathrm{du}$ gouvernement sans que cela n'amène l'opposition à offrir une alternative de gouvernement. En somme, il ne reste plus qu'à savoir si cette nouvelle configuration du

15 Le président du Parlement a hérité d'une fonction dévolue traditionnellement au roi. Face à l'affaiblissement de son rôle à l'issue de l'adoption de la Constitution de 1974, le roi a confié au président du Riksdag le soin de mener ces négociations à sa place.

16 Le Gouvernement Löfven II n'est composé que des sociaux-démocrates et des Verts ce qui porte son soutien parlementaire à cent seize députés sur trois cent quarante-neuf.

17 P. LAVAUX, «Aspects du parlementarisme scandinave : le système de la confiance présumée », RDP, 4-1987, p. 939

18 Stefan Löfven a reçu 115 voix pour, 153 voix contre et 77 abstentions. Si la majorité constitutionnelle pour empêcher sa nomination n'est pas atteinte, il n'obtient pas une majorité favorable à son élection. V. Voterignsprotokoll2018/19 : Statsministeromröstning, 18 janvier 2019.

19 On assiste depuis septembre 2018 a une situation originale dans laquelle un gouvernement et un président du Parlement issus du même renouvellement électoral ayant conduit à l'installation d'une nouvelle législature, appartiennent à des sensibilités politiques différentes et concurrentes sous la présente législature. 
pouvoir gouvernemental peut s'adapter aux institutions parlementaires suédoises ayant maintes fois prouvé leur élasticité. 